\title{
Reflexões sobre os desafios e perspectivas no enfrentamento da obesidade no âmbito
}

\section{da atenção básica no Brasil}

\author{
Reflections on the challenges and prospects in fighting obesity in the context of primary health care
}

in Brazil

Reflexiones sobre los desafíos y perspectivas en la lucha contra la obesidad en el ámbito de la atención primaria en Brasil

Layonne de Sousa Carvalho

ORCID: https://orcid.org/0000-0002-7795-035X Universidade Federal do Piauí, Brasil

E-mail: layonnesc@hotmail.com

Claudiane Batista de Sousa

ORCID: https://orcid.org/0000-0003-0034-3129

Universidade Federal do Piaú, Brasil

E-mail: claudiane_1405@hotmail.com

Jéssica Batista Beserra

ORCID: https://orcid.org/0000-0001-9420-0166

Universidade Federal do Piauí, Brasil

E-mail: jessica_beserra@hotmail.com

Sarah de Melo Rocha Cabral

ORCID: https://orcid.org/0000-0002-4926-6876

Universidade Federal do Piauí, Brasil

E-mail: sarahmelo_9@hotmail.com

Osmar de Oliveira Cardoso

ORCID: https://orcid.org/0000-0001-6093-7629

Universidade Federal do Piauí, Brasil

E-mail: osmar.cardoso@gmail.com

Marize Melo dos Santos

ORCID: https://orcid.org/0000-0003-0699-8062

Universidade Federal do Piauí, Brasil

E-mail: marizesantos@ufpi.edu.br

\begin{abstract}
Resumo
Objetivou-se realizar uma reflexão voltada aos desafios e perspectivas no enfrentamento da obesidade na atenção básica. A obesidade consiste em grave problema de saúde pública, considerada epidemia mundial. Sua prevenção no âmbito da atenção primária à saúde exige mobilização de equipe multiprofissional qualificada e integrada. Os profissionais veiculam estratégias de educação alimentar e nutricional direcionadas aos indivíduos, famílias e comunidades; contribuem para o fortalecimento do direito à alimentação saudável e estabelecem vínculos com os usuários do sistema de saúde. $\mathrm{O}$ atendimento no nível primário de assistência torna-se momento oportuno para mudanças de hábitos alimentares. Dificuldades relacionadas a esta temática são recorrentes na prática profissional dos nutricionistas, o que exige mudança no olhar da gestão pública para maior suporte à assistência nutricional. Recomenda-se a capacitação dos profissionais da atenção básica envolvidos nos processos de cuidado, a fim de reformularem as suas práticas de formação e adotar novos conceitos e práticas no processo de trabalho a fim de melhorar a qualidade de vida da população, promovendo saúde e prevenindo a obesidade e seus agravos.
\end{abstract}

Palavras-chave: Obesidade; Atenção primária à saúde; Saúde pública; Educação alimentar e nutricional.

\begin{abstract}
The objective was to conduct a reflection focused on the challenges and perspectives in the fight against obesity in primary care. Obesity is a serious public health problem, considered a worldwide epidemic. Its prevention in the context of primary health care requires the mobilization of a qualified and integrated multidisciplinary team. The professionals carry out food and nutrition education strategies aimed at individuals, families and communities; they contribute to the strengthening of the right to healthy eating and establish links with users of the health system. Care at the primary care level becomes an opportune moment for changes in eating habits. Difficulties related to this theme are recurrent in the professional practice of nutritionists, which requires a change in the view of public management for greater support to nutritional assistance. It is recommended the training of professionals involved in the primary care process in order to
\end{abstract}


reformulate their training practices and adopt new concepts and practices in the work process in order to improve the population's quality of life by promoting health and preventing obesity and its problems.

Keywords: Obesity; Primary health care; Public health; Food and nutrition education.

\section{Resumen}

El objetivo fue llevar a cabo una reflexión centrada en los desafíos y las perspectivas para enfrentar la obesidad en la atención primaria. La obesidad es un grave problema de salud pública, considerada una epidemia mundial. Su prevención en el contexto de la atención primaria de salud requiere la movilización de un equipo multidisciplinario calificado e integrado. Los profesionales llevan a cabo estrategias de educación alimentaria y nutricional dirigidas a individuos, familias y comunidades; contribuyen al fortalecimiento del derecho a una alimentación saludable y establecen vínculos con los usuarios del sistema de salud. La atención en el nivel de atención primaria se convierte en un momento oportuno para los cambios en los hábitos alimenticios. Las dificultades relacionadas con este tema son recurrentes en la práctica profesional de los nutricionistas, lo que requiere un cambio en la visión de la gestión pública para un mayor apoyo a la asistencia nutricional. Se recomienda la capacitación de profesionales involucrados en los procesos de atención primaria para reformular sus prácticas de capacitación y adoptar nuevos conceptos y prácticas en el proceso de trabajo para mejorar la calidad de vida de la población, promover la salud y prevenir obesidad y sus problemas.

Palabras clave: Obesidad; Atención primaria de salud; Salud pública; Educación alimentaria y nutricional.

\section{Introdução}

A obesidade é uma doença crônica multifatorial condicionada por fatores biológicos, ambientais, socioeconômicos, psicossociais e culturais. Esse agravo é um problema de saúde pública com alta prevalência no Brasil e no mundo, com repercussão no aumento da incidência de outras doenças crônicas não transmissíveis, como diabetes, câncer e doenças cardiovasculares, contribuindo para o aumento da mortalidade da população (Henriques et al., 2018; Mensorio \& Costa Júnior, 2016).

Devido à alta prevalência da obesidade e às comorbidades associadas, é essencial a organização do serviço de saúde para atender a essa demanda de maneira organizada e qualificada (Brasil, 2014a). Desta forma, a obesidade tem se tornado objeto de políticas públicas no Brasil nos últimos 15 anos, e o Ministério da Saúde, no âmbito do Sistema Único de Saúde (SUS), é o principal propositor de ações voltadas para sua prevenção e tratamento (Dias et al., 2017). Essas ações são realizadas em determinados ambientes da atenção à saúde que ofertam serviços por meio de uma produção singular do cuidado. Dentre esses espaços podem-se citar: as Unidades Básicas de Saúde (UBS), as unidades ambulatoriais especializadas, os Centros de Atenção Psicossocial (CAPS), as residências terapêuticas, entre outros (Brasil, 2014b).

Destacam-se ainda as ações de alimentação e nutrição na Atenção Básica à Saúde, principalmente após a inserção do nutricionista, em 2008, nas equipes dos Núcleos de Apoio à Saúde da Família (NASF), equipes multidisciplinares que visam garantir o matriciamento das ações de Alimentação e Nutrição às equipes de atenção básica. Além disso, no ano de 2011, foi lançado o Programa Academia da Saúde, que tem como objetivo contribuir para a promoção da saúde e hábitos de vida saudáveis para população a partir da implantação de polos com infraestrutura adequada e profissionais qualificados, potencializando as ações de cuidados individuais e coletivos na atenção básica para o enfrentamento da obesidade (Coelho \& Verdi, 2015; Sá, et al., 2016).

Nessa perspectiva, a assistência primária à saúde é a porta de entrada do sistema de saúde, sendo local privilegiado para as ações de cuidado integral da obesidade, com ênfase no manejo alimentar e nutricional de indivíduos, famílias e comunidades, por conta da proximidade dos profissionais de saúde ao cotidiano de vida da população. Desta forma, torna-se o nível de atenção apropriado para promoção da saúde e prevenção de agravos como a obesidade.

Diante da abrangência e o escopo das ações realizadas na atenção primária à saúde, destaca-se a importância dos profissionais nutricionistas para a efetividade das ações realizadas na prevenção e controle da obesidade, uma vez que alia conhecimentos sobre práticas educativas em nutrição, prescrição dietoterápica e técnica dietética na construção das ações relativas à prevenção de doenças crônicas e cuidado integral ao indivíduo obeso. 


\section{Metodologia}

Tendo em vista a alta prevalência da obesidade no Brasil e a Atenção Primária à Saúde como forte aliada para o seu enfrentamento, este artigo teve como objetivo trazer reflexões voltadas aos desafios e perspectivas no enfrentamento da obesidade no âmbito da atenção básica por meio de uma investigação teórica. Trata-se de um estudo teórico-reflexivo com uma abordagem qualitativa utilizando-se principalmente documentos oficiais do Ministério da Saúde e artigos brasileiros publicados nos últimos 10 anos referenciando a temática (Ludke \& Andre, 2013; Minayo, 2006).

\section{Resultados e Discussão}

\section{Ações, desafios e perspectivas do enfrentamento da obesidade no contexto dos Centros de Atenção Psicossocial}

Os Centros de Atenção Psicossocial (CAPS) constituem-se locais de referência de promoção de saúde e tratamento de indivíduos com transtornos mentais, oferecendo serviços comunitários, ambulatoriais e regionalizados, ao passo que reafirmam a convergência das ações relativas à saúde coletiva e a saúde mental de seus usuários no âmbito do Sistema Único de Saúde (Leal \& Antoni, 2013).

Na perspectiva da saúde integral do indivíduo com transtorno mental atendido nos CAPS, o enfrentamento da obesidade destaca-se como um desafio na prática de profissionais da saúde mental, uma vez que estudos epidemiológicos têm constatado relação entre o maior acúmulo de gordura, evidenciado pelo alto Índice de Massa Corporal (IMC), com a ocorrência de transtornos psiquiátricos (Kengeriski et al., 2014).

Observa-se que o ganho de peso dos pacientes psiquiátricos se dá, em parte, pela inatividade física, alterações neuroendócrinas decorrentes dos próprios quadros e pelo uso de alguns psicofármacos, como antidepressivos tricíclicos, inibidores de monoaminoxidase (IMAOS) e lítio. Entre as hipóteses para aumento de peso causado pelo uso de psicofármacos estariam o aumento de compulsões alimentares ou ainda alterações serotoninérgicas (Aratangy; Kachani; Codás, 2010).

O cuidado nutricional de indivíduos com transtornos mentais com obesidade é complexo visto que os medicamentos diferem bastante no que diz respeito ao seu efeito sobre o peso e nas diferenças individuais de metabolismo. Assim, exige-se o acompanhamento e registro de alterações da doença e dos diferentes tipos e doses de antipsicóticos utilizados no tratamento para melhor entender e tratar o ganho de peso excessivo nesses pacientes (Zortea et al., 2010).

Nesse contexto, as ações realizadas por nutricionistas na atenção primária à saúde como a avaliação do perfil antropométrico e do consumo alimentar, juntamente com ações educativas e de intervenção nos hábitos alimentares e de estilo de vida são relevantes na identificação e diagnóstico precoce de complicações associadas ao uso de antipsicóticos pelos usuários. Desta maneira, essas ações são importantes para a melhoria da qualidade de vida e autonomia dos usuários do CAPS (Burlin et al., 2016).

Os Centros de Atendimento Psicossocial podem ser cenários para a ação de aprimoramento e promoção da saúde por serem espaços de encontros e de trocas que fortalecem a articulação da ação e integração entre os profissionais e equipes do serviço, priorizando a interdisciplinaridade e o olhar atento ao usuário, nas suas múltiplas dimensões, incluindo a questão da obesidade.

\section{Ações, desafios e perspectivas no enfrentamento da obesidade no contexto do Núcleo de Apoio à Saúde da Família}

O nutricionista é um dos profissionais que compõem a equipe do Núcleo de Apoio a Saúde da Família (NASF), que tem entre as principais atribuições o desenvolvimento de ações de promoção de práticas alimentares saudáveis, cabendo a este profissional atuar de forma efetiva sobre os agravos e distúrbios alimentares e nutricionais que acometem a população em todos os estágios de vida. Sabe-se que a elevada prevalência de obesidade tem sido associada diretamente ao estilo de vida dos indivíduos, destacando-se aqueles de caráter comportamental, como os hábitos alimentares e o sedentarismo (Rech et al., 2016; 
Almeida et al., 2017).

As ações de prevenção e controle da obesidade devem oferecer apoio aos indivíduos, de modo a incentivá-los a adotarem hábitos de vidas saudáveis que resultem na manutenção e recuperação do peso. Para isso, é importante a articulação entre saberes, serviços e instituições da Rede de Atenção à Saúde (RAS), em busca da intersetorialidade e atenção multidisciplinar, por meio de parcerias, vínculos e assim, substituir a atenção centrada no ser humano por aquela que promova qualidade de vida para a população (Sowek, 2020).

Por tratar-se de um problema multifatorial e de consequências graves para a população, isso implica em desafios para as equipes de saúde, que exigem aperfeiçoamento nas estratégias de cuidado e prevenção do excesso de peso e no próprio modelo de cuidado a esses indivíduos, que muitas vezes resulta em tratamentos ineficazes (Cori et al., 2015). Um dos principais pontos que requer reorganização do modelo da atenção é a abordagem profissional frente a essa questão, visto que, ainda é notável uma compreensão limitada do excesso de peso e a adoção de abordagem biologicista, o que afeta diretamente a eficácia do cuidado realizado pelos profissionais (Paiva, 2016).

Para o enfrentamento desse cenário é necessário que as ações e políticas de controle desse quadro proporcionem ambientes estimulantes e que mantenham modelos de atenção adequados para a alimentação, atividade física, que possam ir além da informação e educação (Rech et al., 2016). A organização da atenção e gestão do SUS ainda se caracteriza pela fragmentação de serviços, programas, ações e práticas clínicas, o que torna inadequada e ineficiente para enfrentar os desafios impostos pela ascensão da obesidade; portanto, é preciso avançar na qualificação da atenção e da gestão em saúde, enquanto protagonistas desse processo, formando-se assim uma rede de apoio integrada e articulada que envolva todos os níveis de atenção (Brasil, 2014b).

Entre os principais desafios para o enfrentamento da obesidade na Atenção Primária em Saúde (APS) encontrados em uma revisão realizada por Almeida et al., (2017) destacam-se a prevalência de obesidade entre as populações de baixa renda, a baixa capacitação dos profissionais de saúde, a escassez de promoção da saúde na APS, a dificuldade em avaliar a efetividade das ações de alimentação e nutrição nas escolas, e a qualidade das informações nos prontuários e protocolos de nutrição nas UBS.

Dessa forma, diante das experiências vivenciadas no Núcleo de Apoio da Saúde da Família, é possível perceber que as instituições e profissionais ainda são focados na realização de atendimentos individuais e prescritivos, sem a realização do acompanhamento longitudinal do indivíduo obeso e apoio multidisciplinar. Além disso, existem outras barreiras para a realização de ações efetivas e contínuas, como a insuficiência de espaços físicos para as práticas de atividades de educação em saúde, ineficiente estrutura física para os atendimentos, equipamentos de avaliação reduzidos e precários, a ausência de psicólogos, além do número insuficiente de profissionais da área de Nutrição e Educação Física, o que constituem fatores limitantes no processo de implementação da linha de cuidado ao indivíduo com excesso de peso na Atenção Básica.

Vale ressaltar ainda que, a realização de trabalho em equipe ainda constitui um desafio da atenção nutricional realizada na Atenção Básica, tendo em vista que a obesidade é um problema multifatorial, portanto, demanda a realização de trabalhos em equipes multiprofissionais, visando atender à complexidade que envolve esta problemática.

Outros desafios ainda incluem a necessidade de apoio institucional efetivo, organização das ações de referência e contrarreferência (visto que a continuidade do tratamento é necessária), além da participação de uma equipe multidisciplinar comprometida com a resolução do problema, deixando de ser um ato isolado para uma ação que mobiliza todos os pontos da rede (Brasil, 2014b).

Nota-se a importância da ampliação das ações por meio de parcerias com outros setores e profissionais, que podem contribuir para o fortalecimento e abrangência dessas no âmbito do sistema de saúde (Paiva, 2016). Essas ações intersetoriais podem incluir órgãos pontuais para o desenvolvimento de ações de alimentação, nutrição e atividade física, a exemplo, 
Secretarias Municipais/Estaduais de Esporte e Lazer, Centros de Referência da Assistência Social (CRAS), Associações de Agricultores Familiares e Secretarias de Administração e Educação, permitindo que as ações sejam mais ordenadas e resolutivas (Almeida et al., 2017).

Além disso, ressalta-se a relevância da gestão dos serviços de saúde e dos profissionais na prevenção e controle dessa comorbidade, fazendo-se necessário criar, organizar, monitorar e avaliar ações de intervenções, visando o alcance do tratamento adequado. Para tanto, torna-se imprescindível incentivar a realização de ações de maneira integrada, uma vez que esse processo é fundamental no manejo da obesidade.

Dessa forma, é fundamental a realização da gestão participativa dos responsáveis pelos serviços de saúde e profissionais, com atenção voltada para a prevenção e controle desta comorbidade, tendo em vista a importância da organização, monitoramento contínuo e avaliação das ações de intervenções, visando o alcance do tratamento adequado.

\section{Ações, desafios e perspectivas no enfrentamento da obesidade no contexto do Programa Academia da Saúde}

O Programa Academia da Saúde teve início em 2011, por meio da Portaria no 719/GM/MS, com o objetivo promover práticas corporais e atividade física, promoção da alimentação saudável e educação em saúde, além de contribuir para produção do cuidado e de estilos de vida saudáveis da população. Seus polos são dotados de infraestrutura, equipamentos e profissionais qualificados (Brasil, 2014b).

Este programa é respaldado pelos princípios e diretrizes da Lei Orgânica da Saúde (Lei nº 8.080/1990), pelas Políticas Nacionais de Promoção da Saúde (Portaria n 2.446, de 11 de novembro de 2014) e de Atenção Básica (Portaria nº 2.436 , de 21 de setembro de 2017) (Brasil, 2018). Desta maneira, busca integrar as ações programáticas e de demanda espontânea; articulando as ações de promoção à saúde, prevenção de agravos, vigilância à saúde, tratamento e reabilitação, e à ampliação da autonomia dos usuários e coletividades; trabalhando de forma multiprofissional, interdisciplinar e em equipe (Brasil, 2012).

Ressalta-se que a Portaria $n^{\circ} 2.681$, de 07 de novembro de 2013, estabelece oito eixos em torno dos quais as atividades dos polos das academias de saúde devem contemplar: práticas corporais e atividades físicas, promoção da alimentação saudável, mobilização da comunidade, educação em saúde, práticas artísticas e culturais, produção do cuidado e de modos de vida saudável, práticas integrativas e complementares, e planejamento e gestão (Brasil, 2014b). Tais ações podem ser ferramentas úteis no manejo e controle de doenças crônicas com a obesidade, e, portanto, devem estar implementadas na execução efetiva do programa.

Em geral, na prática, é possível salientar que as atividades realizadas para o controle e prevenção da obesidade são atendimentos individualizados, por demanda espontânea e por encaminhamentos das equipes saúde da família (e-SF), CAPs e NASF. Além disso, são realizadas atividades físicas como aeróbica, funcional, alongamentos e caminhadas, orientadas e monitoradas por profissionais de educação física; bem como atendimentos coletivos, rodas de conversa, encontros de grupo de usuários obesos, realização de antropometria e palestras educativas, podendo ser conduzidos por nutricionistas, profissionais de educação física, fisioterapeutas e psicólogos vinculados ou não ao programa.

A linha de cuidado do indivíduo obeso prevê a organização da vigilância alimentar e nutricional, a partir da avaliação do peso e da estatura e a avaliação do consumo alimentar; realizações de ações em grupos; promoção de práticas corporais e integrativas e atividade física no espaço da Academia da saúde; ações de promoção da alimentação adequada e saudável; definição de plano de metas individuais, prescrição dietética e avaliação periódica dos usuários (Brasil, 2014c).

Por meio de experiência na execução do programa é possível observar que há uma demanda considerável de usuários com doenças crônicas, representada especialmente pela população de obesos, hipertensos e diabéticos, maioria do sexo feminino. O cotidiano de trabalho revela aumento dos casos de indivíduos com excesso de peso, relacionados, na maioria dos casos, às inadequações alimentares, excesso de consumo de alimentos ultraprocessados e resistência quanto à adesão aos programas de 
reeducação alimentar. Muitos pacientes relatam dificuldades no processo de emagrecimento ou controle do peso por falta de apoio de amigos/ familiares e até mesmo por aderirem a dietas bastante restritivas e indicadas por entes próximos.

O programa possibilita a avaliação e elaboração de um diagnóstico do estado nutricional da população ao passo que se torna espaço de produção e vivências para construção coletiva e individual de hábitos saudáveis. Na perspectiva do enfrentamento da obesidade, é imprescindível reconhecer a importância de conhecer o histórico alimentar do paciente, bem como levar em consideração seus hábitos regionais, crenças, saberes e vivências no intuito de melhor atender suas necessidades e expectativas. Desta forma, a promoção da alimentação adequada e saudável no âmbito do Programa Academia da Saúde deve favorecer o empoderamento do usuário de forma ativa como protagonista de sua própria mudança.

O nutricionista como profissional referência para a promoção de práticas alimentares saudáveis na rede básica de saúde, deve abranger não apenas o atendimento individual, mas buscar formas de atender o coletivo, envolvendo os usuários de forma ativa. Os grupos terapêuticos envolvendo indivíduos obesos possuem o intuito de prevenir e controlar os casos de sobrepeso e obesidade, bem como reduzir as comorbidades associadas ao excesso ponderal. São oportunidades para implementação de abordagem cognitiva-comportamental na construção coletiva do conhecimento em alimentação e nutrição.

É relevante destacar a importância das atividades coletivas e socialização de conhecimentos e experiências entre os envolvidos, bem como da busca ativa de usuários com perfil antropométrico que caracterize o excesso de peso. No âmbito da nutrição, deve-se priorizar temáticas que envolvam a promoção de ações de segurança alimentar e nutricional e de educação alimentar e nutricional, contextualizando com a realidade local, bem como estímulo à prática de atividade física regular e adesão às mudanças nos hábitos alimentares, com valorização da culinária regional. Para tanto, faz-se necessário o suporte de uma equipe multiprofissional, para permanente socialização de experiências, afetos e conhecimentos entre os envolvidos.

Os desafios perpassam desde a desvalorização profissional até a limitação de recursos financeiros e materiais necessários para a execução de atividades cotidianas e imprescindíveis para a boa efetividade de intervenções educativas para o controle da obesidade. Além disso, reforça-se a necessidade de maior envolvimento de setores econômicos, sociais e culturais, uma vez que a obesidade é de complexa etiologia multifatorial, e desta forma, fatores socioeconômicos podem consistir nas principais dificuldades encontradas para o seu enfrentamento.

\section{Considerações Finais}

Recomenda-se a capacitação dos profissionais envolvidos nos processos de cuidado da $\mathrm{AB}$, a fim de reformularem as suas práticas de formação e adotar novos conceitos e práticas no processo de trabalho. Propõe-se ações educativas no âmbito da nutrição, organizadas para que funcionem de forma harmônica e integrada aos demais serviços da atenção básica, instigando a veiculação de conhecimentos em alimentação e nutrição dentro e fora dos programas para melhoria da qualidade de vida da população, promovendo saúde e prevenindo a obesidade e seus agravos.

A compreensão da obesidade de forma ampla consiste em um desafio no contexto atual nas propostas de trabalho da atenção básica brasileira. Por envolver uma gama de fatores determinantes e condicionantes, demanda ações resolutivas e empoderamento da temática pelos envolvidos, usuários do sistema e profissionais de saúde, forma de garanta a corresponsabilização pelas ações a serem realizadas. Portanto, estudos observacionais devem ser realizados em torno da temática com enfoque nos determinantes e condicionantes de saúde que envolvam a obesidade, na efetividade das ações realizadas na atenção básica, bem como sobre os saberes e as práticas dos profissionais de saúde envolvidos no processo de enfrentamento da obesidade.

Apoio: Chamada CNPq/MS/SAS/DAB/CGAN No 26/2018. 
Research, Society and Development, v. 10, n. 7, e6810716331, 2021

(CC BY 4.0) | ISSN 2525-3409 | DOI: http://dx.doi.org/10.33448/rsd-v10i8.16331

\section{Referências}

Almeida, L. M., Campos, K. F. C., Randow, R., \& Almeida, V. (2017). Estratégias e desafios da gestão da atenção primária à saúde no controle e prevenção da obesidade. Rev. Gestão \& Saúde, 8, 114-139.

Aratangy, E. W., Kachani, A. T., Cordas, T. A. (2010). Tratamento Medicamentoso e Ganho de Peso. in Cordás TA, Kachani AT. Nutrição em Psiquiatria. Porto Alegre: Artmed, 77-89.

Brasil. Ministério da Saúde (2014A). Secretaria de Atenção à Saúde. Departamento de Atenção Básica. Estratégias para o cuidado da pessoa com doença crônica: obesidade. Brasília: Ministério da Saúde.

Brasil. Ministério da Saúde. Organização Pan-Americana da Saúde (2014B). Perspectivas e desafios no cuidado às pessoas com obesidade no SUS: resultados do Laboratório de Inovação no manejo da obesidade nas Redes de Atenção à Saúde. Brasília: Ministério da Saúde.

Brasil. Ministério da Saúde. Secretaria de Atenção à Saúde. Departamento de Atenção Especializada e Temática. Coordenação-Geral de Atenção às Pessoas com Doenças Crônicas (2014C). Organização Regional da Linha de Cuidado do Sobrepeso e da Obesidade na Rede de Atenção à Saúde das Pessoas com Doenças Crônicas - Manual Instrutivo. Brasília: Ministério da Saúde.

Brasil. Ministério da Saúde. Secretaria de Atenção à Saúde. Departamento de Atenção Básica. (2012). Política Nacional de Atenção Básica. Brasília: Ministério da Saúde.

Brasil. Ministério da Saúde. Secretaria de Atenção à Saúde. Departamento de Atenção Básica. (2018). Programa Academia da Saúde: caderno técnico de apoio a implantação e implementação. Brasília: Ministério da Saúde.

Burlin, A. C., Volpato T., Lopes, E., Ariotti, A. P., Gazzi, L., Cenci, F. M., Remor, A. P., Barcelos, A. L. V., Rossoni, C. (2016). Avaliação nutricional de usuários atendidos em um Centro de Atenção Psicossocial (CAPS). BRASPEN J, 31(3), 226-316.

Coelho, C. S. \& Verdi, M. I. M. (2015). Políticas e programas de atividade física: uma crítica à luz da promoção da saúde. Sau. \& Transf. Soc., 6(3), 96-108.

Cori, G. C., Petty, M. C. B., \& Alvarenga, M. S. (2015). Atitudes de nutricionistas em relação a indivíduos obesos - um estudo exploratório. Ciênc. saúde coletiva, 20(2), 565-576.

Dias, P. C., Henriques, P., Anjos, L. A., \& Burlandy, L. (2017). Obesidade e políticas públicas: concepções e estratégias adotadas pelo governo brasileiro. Cad. Saúde Pública, 33(7), 1-12.

Henriques, P., O’Dwyer, G., Dias, P. C., Barbosa, R. M. S., \& Burlandy, L. (2018). Políticas de Saúde e de Segurança Alimentar e Nutricional: desafios para o controle da obesidade infantil. Ciênc. saúde coletiva, 23(12), 4143-4152.

Kengeriski, M. F., Oliveira, L. D., Escobar, M., Delgado, V. B. (2014). Estado nutricional e hábitos alimentares de usuários em Centro de Atenção Psicossocial de Porto Alegre, Brasil. Clin Biomed Res., 34(3), 253-259.

Leal, B. M. \& Antoni, C. (2013). Os Centros de Atenção Psicossocial (CAPS): estruturação, interdisciplinaridade e intersetorialidade. Aletheia, 40: 87-101.

Ludke, M. \& Andre, M. E. D. A. (2013). Pesquisas em educação: uma abordagem qualitativa. São Paulo: E.P.U.

Mensorio, M. S., Costa Junior, A. L. (2016). Obesidade e estratégias de enfrentamento: o que destaca a literatura? Psicol. saúde doenças, 17(3), 468-482.

Minayo, M. C. S. O desafio do conhecimento: pesquisa qualitativa em saúde. $9^{\text {a }}$ ed. São Paulo: EDUC; 2006.

Paiva, A. L. S. (2016). O nutricionista e o cuidado ao indivíduo com excesso de peso na atenção básica do Distrito Federal. Dissertação (Mestrado em Nutrição Humana) - Universidade de Brasília, Brasília,111p.

Rech, D. C., Borfe, L., Emmanouilidis, A., Garcia, E. L., Krug, S. B. F. (2016). As políticas públicas e o enfrentamento da obesidade no Brasil: uma revisão reflexiva. Rev. Epidemiol. Controle Infecç., 6, 192-202.

Sá, G. B. A. R., Dornelles, G. C., Cruz, K. G., Amorim, R. C. A., Andrade, S. S. C. A., Oliveira, T. P., Silva, M. M. A., Malta, D. C., \& Souza, M. F. M. (2016). O Programa Academia da Saúde como estratégia de promoção da saúde e modos de vida saudáveis: cenário nacional de implementação. Ciênc. saúde coletiva, 21(6),1849-1860.

Sowek, L. E. (2020). Tratamento de adultos obesos usuários do SUS: uma proposta interdisciplinar. Dissertação (Mestrado em Ciências da Saúde) Universidade Estadual de Ponta Grossa, Ponta Grossa, 110p.

Zortea, K., Guimarães, L. R., Gama, C. S., \& Belmonte-de-Abreu, P. S. (2010). Estado nutricional de pacientes com esquizofrenia frequentadores do Centro de Atenção Psicossocial (CAPS) do Hospital de Clínicas de Porto Alegre. J. bras. psiquiatr., 59(2),126-130. 